\title{
Effect of Exercise on Some Haemostatic Parameters Among Students in a Tertiary Educational Institution in Nigeria
}

\author{
Benjamin Onyema Eledo ${ }^{1,2}$, Reward Igwe ${ }^{1}$, Okezie Caleb Okamgba ${ }^{3}$, Sylvester Chibueze Izah ${ }^{4}$ * \\ ${ }^{1}$ Department of Medical Laboratory Science, Faculty of Health Sciences, Madonna University, Elele, Nigeria \\ ${ }^{2}$ Haematology and Blood Transfusion Department Federal Medical Centre, Yenagoa, Nigeria \\ ${ }^{3}$ Department of Medical Laboratory Science, Abia State University, Uturu, Nigeria \\ ${ }^{4}$ Department of Biological Sciences, Faculty of Science, Niger Delta University, Wilberforce Island, Nigeria
}

Email address:

chivestizah@gmail.com (S. C. Izah)

${ }^{*}$ Corresponding author

\section{To cite this article:}

Benjamin Onyema Eledo, Reward Igwe, Okezie Caleb Okamgba, Sylvester Chibueze Izah. Effect of Exercise on Some Haemostatic Parameters Among Students in a Tertiary Educational Institution in Nigeria. American Journal of Health Research.

Vol. 5, No. 5, 2017, pp. 145-148. doi: 10.11648/j.ajhr.20170505.15

Received: August 13, 2017; Accepted: August 28, 2017; Published: September 18, 2017

\begin{abstract}
This study investigated the effect of exercise on some haemostatic parameters among students from a tertiary institution in Nigeria. Fifty four individuals participated in this study, comprising 28 males and 26 females. Blood was collected from the participants before and after the rigorous exercise and the prothrombin time (PT), activated partial thromboplastin time (APTT) and platelets count (PLT) were analyzed using standard methodology. Results showed mean values before and after the exercise were 217.31 and $238.27 \times 10^{9} / \mathrm{L}$ for female, 228.75 and $240.18 \times 10^{9} / \mathrm{L}$ for male respectively (platelets counts), 14.25 and 12.08 seconds for female, 14.29 and 12.35 seconds for male respectively (prothrombin time) and 31.71 and 24.29 seconds for female and 30.62 and 23.43 seconds for male respectively (activated partial thromboplastin time). Statistically, there was significant variation $(\mathrm{P}<0.001)$ before and after exercise for PT, APTT, and no significant difference $(\mathrm{P}>0.05)$ for PLT count before and after exercise. PT and APTT showed significant decline after the exercise indicating activation of the coagulation cascade which may lead to hypercoagulability predisposing the subjects to an increased risk of cardiovascular derangement; while PLT showed no variation suggesting no risk of thrombocytopenia or thrombocytosis.
\end{abstract}

Keywords: Exercise, Haemostatic Indices, Prothrombin Time, Activated Partial Thromboplastin Time, Blood Platelets

\section{Introduction}

Exercise is any action that involves part or full body to maintain physical fitness. Exercise is essential for the maintenance of the various organs and tissues at optimal level especially at regular and moderate rate. Exercise is also encouraged as a remedy for some disease conditions. For instance, Lekakis et al. [1] reported that exercise is often recommended for the treatment of patients with arterial hypertension. Furthermore, exercise is also essential in preventing and/ or reducing some health conditions such as obesity which is a risk factor for different cardiovascular diseases. Regular exercise could also boost the immune system; keep fitness and prevent over ageing.

Exercise often leads to physiological stress in the human body. According to Bhatti and Shaikh [2], exercise could lead to variation in chemical (hormonal) and cellular system, increased blood pressure, body temperature and oxygen intake. This characteristic often varies in individuals depending on type and duration of exercise, climate, physical body status, nutrition etc [2].

Authors have variously reported the role of exercise in human health based on blood related parameters. Studies have shown that exercise induces immune response, leading to 
leukocytosis that is quantitatively likened to be a response against physiological insults to the immune system [2]. Bhatti and Shaikh [2] reported no significant variation among individuals with mean age of 23 before and after jogging for 30 minutes with regard to haemoglobin, total white blood cells count, systolic and diastolic blood pressure, erythrocyte sedimentation rate. Benoni et al. [3] reported that physical exercise (10 minutes on a cycle ergometer at a heart rate of 150 beats/ minutes) leads to significant elevation in total leucocyte, lymphocyte and neutrophil concentrations and lower serum iron and ferritin concentrations in active subjects.

Exercise induces modification in blood haemostasis [4. 5]. Typically, the haemostatic system aid in the maintenance of blood in a fluid state, stop bleeding due to vascular injury by development of a haemostatic plug which are destroyed slowly during healing [6]. Several activation parameters of blood exist. Some of these include coagulation viz: prothrombin time, activated partial thromboplastin time, fibrinogen, D-Dimers, prothrombin fragments $1+2$, thrombin-antithrombin III complex [and factors VII, VIII and XII), platelet viz: platelet count, platelet factor 4 and $\beta$ thromboglobulin and fibrinolysis viz: plasmin- $\mathrm{a}_{2}$ antiplasmin complex [1]. The authors further reported endothelial function viz: soluble thrombomodulin and von Willebrand factor as some parameters that play essential role in blood function.

Platelets play essential role in human body. Some of the roles include blood clotting, prevention of excess bleeding, haemorrhage and leakage of red blood cell [7]. When the platelet counts are low it could lead to increased bleeding. Low platelet count often emanates from disease condition such as leukemia (a bone marrow disease), rheumatoid arthritis and lupus (an autoimmune disorders), chemotherapy, kidney dysfunction, viral infections, reactions to blood transfusions, excessive alcohol intake and low levels of vitamin B12 [1].

According to El-Sayed [8], results on platelet aggregation and markers of platelet activation from individual that undergone exercise varies and are conflicting. The author further suggested that short term exercise activates blood coagulation and enhances blood fibrinolysis and the delicate balance between clot formation and clot dissolution is maintained in normal populations [8].

Prothrombin and activated partial thromboplastin time are parameters used to assess coagulation properties of blood as such they are essential in assessing risk of development of bleeding complications [9]. Peterson and Gottfried [10], Lippia et al. [11] also reported that prothrombin and activated partial thromboplastin time are essential parameters in assessing haemostatic functions.

According to Abdullah [6], vascular, platelet and coagulation factors also play vital role in the development of coronary artery disease. Therefore, this present study aimed at evaluating the effects of exercise on some haemostatic parameters with special focus on prothrombin time, activated partial thromboplastin time and platelets count on students of a tertiary institution in Nigeria.

\section{Materials and Methods}

\subsection{Study Area}

River state is one of the states in the Niger Delta region of Nigeria. The state has population of over 6 million. The state shares boundary with neighboring Niger Delta states including Bayelsa in the Western and southern region, Abia in the East and Atlantic Ocean in the Southernmost part. Several economic activities are carried out in the area including fishing, farming, business and several industries and civil servant. Emuoha local government is one of the areas in River state. The climatic region of the region is characterized by relative humidity and temperature of $50-$ $95 \%$ and $28 \pm 6^{\circ} \mathrm{C}$ all year round. Two known predominant seasons viz: wet (April to October) and dry (November to March of the following year). Recently, the seasons appear to be shifting from known conventional period of 5 and 7 months dry and wet season respectively.

\subsection{Selection Criteria for Subjects}

Inclusion Criteria: Subjects for this research were male and female students of Madonna University, Elele campus, River state who engage in rigorous exercises every Monday and Tuesdays. The exercise involved series of jogging and a marathon race that lasted for two hours. A Total of fifty four (54) students participated in this study which comprised of males (28) and females (26) within the age of $18-26$ years. Blood samples were collected before and after the exercise.

Exclusion criteria: Pregnant women and individuals with known cases of hepatitis, diabetes, tuberculosis, cardiovascular diseases, bleeding and blood clotting disorders were excluded.

\subsection{Blood Collection}

A standard venipuncture technique was used to collect $5 \mathrm{ml}$ of blood from each subject from the anticubital or dorsal vein. Approximately $2.25 \mathrm{mls}$ was dispensed into plastic tube containing $0.25 \mathrm{ml}$ of trisodium citrate to be used for prothrombin time and activated partial thromboplastin time analysis, while the remaining $2.75 \mathrm{mls}$ of blood was dispensed into dipotassium EDTA bottles containing $1.5 \mathrm{mg} / \mathrm{ml}$ of blood of the anhydrous salt which was used for the platelets count determination.

\subsection{Laboratory Analysis}

\subsubsection{Prothrombin Time}

Principle: Tissue thromboplastin in the presence of $\mathrm{Ca}^{++}$ activates extrinsic pathway of human blood coagulation cascade. Activation time is proportional to the concentration of individual clotting factors taking part in the coagulation cascade. When thromboplastin reagent is added to citrated plasma, clotting cascade is initiated forming gel clot. The time required for clot formation would be prolonged if there is deficiency of factor(s) activity in the extrinsic pathway of the coagulation cycle.

Procedure: Kit supplied by Agappe Diagnostics Switzerland. 
Lot number: 52601003 were used to analyze the Prothrombin time. The PT REAGENT was pre-warmed to $37^{\circ} \mathrm{C}$ for 10 minutes. Then $0.1 \mathrm{ml}$ of plasma was dispensed into test cuvette at $37^{\circ} \mathrm{C}$ and incubated for 3 minutes. Approximately, $0.2 \mathrm{ml}$ of prewarmed PT REAGENT was forcibly added into the test cuvette. The timer was started simultaneously and the time for the first clot to appear was recorded in seconds. The test was done in triplicate and the average time was recorded as the prothrombin time. Same procedure was applied on the blood samples collected after the exercise

\subsubsection{Activated Partial Thromboplastin Time}

Principle: In the presence of calcium ions cephaloplastin activates coagulation factors of intrinsic pathway in plasma leading to clot formation. Clotting time is proportional to the concentration of factors viii, ix, $x i$ and xiii as well as common pathway factors ii, $\mathrm{v}$, and $\mathrm{x}$.

Procedure: Kit supplied by Agappe Diagnostics Switzerland. Lot number: 52602001 were used to analyze the Activated Partial Thromboplastin time. Reagent $1\left(\mathrm{CaCl}_{2}\right)$ and reagent 2 (APTT REAGENT) were pre-warmed at $37^{\circ} \mathrm{C}$. Then $0.1 \mathrm{ml}$ of before/after exercise plasma was dispensed into test cuvettes at $37^{\circ} \mathrm{C} .0 .1 \mathrm{ml}$ of the pre-warmed reagent was added into the test cuvettes, mixed and incubated at $37^{\circ} \mathrm{C}$ for 3 minutes. Then after, $0.1 \mathrm{ml}$ of pre-warmed reagent 1 was forcibly added into the test cuvettes. The timer was started simultaneously and the time for the first clot to appear was recorded in seconds. The test was carried out in triplicate and the average time was recorded as the activated partial thromboplastin time.

\subsubsection{Platelets Count}

This was based on the Cronkite's ammonium oxalate method where the red cells were lysed and the platelets left as highly refractile particles under illumination. A 1:20 dilution of blood was made by adding $0.1 \mathrm{ml}$ of blood to $1.9 \mathrm{ml}$ of diluents (ammonium oxalate). The suspension was well mixed and an improved Neubauer counting chamber covered with cover slip was charged with the suspension and left untouched in the moist petri-dish for about 20 minutes to allow the platelets to settle. The number of platelets in two areas of $1 \mathrm{~mm}$ square were counted using x40 objective. The number of platelets per litre was calculated from the formula:

$$
\text { Platelets }=\frac{\text { Number of cells counted } \mathrm{x} \text { dilution } \times 10^{6}}{\text { volume }(\mu \mathrm{l})}
$$

\subsection{Statistical Analysis}

SPSS software version 20 was used to carry out statistical analysis. Data were presented and mean \pm standard error. The haemostatic values obtained before and after exercise were subjected to " $\mathrm{t}$ " test. Significance level was determined at Pvalue of $<0.05$.

\section{Results and Discussion}

The effect of exercise on some haemostatic parameters in females and males from a tertiary institution in Nigeria is presented in Table 1 and 2 respectively. In the female, the mean platelets were 217.31 and $238.27 \times 10^{9} / \mathrm{L}$ before and after exercise respectively (Table 1). In the male, mean platelets counts were 228.75 and $240.18\left(\times 10^{9} / \mathrm{L}\right)$ before and after exercise respectively (Table 2). Basically, there was no significant variation $(\mathrm{P}>0.05)$ before and after the exercise. The findings showed that exercise apparently enhances platelet counts but were not statistically valid at $\mathrm{P}=0.05$.

Typically, platelet count increases with exercise and this is probably due to a fresh release of platelets from the spleen, bone marrow and lungs [8]. But when the bone marrow is unable to produce platelets lesser than the volume being utilized, it could lead to low platelet count or a condition known as thrombocytopenia [12]. Furthermore, Moore [7] reported that during exercise in healthy persons, tiny injuries to blood vessels and tissues usually occur and the individual will not be able to notice, and it often heals quickly because platelets are available in the region to stop bleeding and permit healing. Therefore, no significant difference $(\mathrm{P}>0.05)$ before and after exercise suggests that the exercise is not vigorous enough to cause cardiovascular health related diseases. According to Wang et al. [13, 14], platelets play a vital role in pathogenesis of cardiovascular diseases condition. Regular exercise reduces the risk of cardiovascular disease while vigorous exercise incites unexpected cardiac health challenge [13].

Table 1. Effect of exercise on some haemostatic parameters in females.

\begin{tabular}{|c|c|c|c|c|}
\hline \multirow{2}{*}{ Parameters } & \multicolumn{2}{|c|}{ Mean \pm standard error } & \multirow{2}{*}{ t-value } & \multirow{2}{*}{ P-value } \\
\hline & Before $(n=26)$ & After $(n=26)$ & & \\
\hline Prothrombin time (PT), secs & $14.25 \pm 0.18$ & $12.08 \pm 0.14$ & 9.426 & 0.000 \\
\hline Activated partial thromboplastin time (APTT), secs & $31.71 \pm 0.58$ & $24.29 \pm 0.27$ & 11.585 & 0.000 \\
\hline Platelets counts $(\mathrm{PLT})\left(\mathrm{x} 10^{9} / \mathrm{L}\right)$ & $217.31 \pm 9.58$ & $238.27 \pm 14.11$ & -1.229 & 0.225 \\
\hline
\end{tabular}

Table 2. Effect of exercise on some haemostatic parameters in males.

\begin{tabular}{|c|c|c|c|c|}
\hline \multirow{2}{*}{ Parameters } & \multicolumn{2}{|c|}{ Mean \pm standard error } & \multirow{2}{*}{ t-value } & \multirow{2}{*}{ P-value } \\
\hline & Before $(n=28)$ & After $(n=28)$ & & \\
\hline Prothrombin time $(\mathrm{PT})$, secs & $14.29 \pm 0.13$ & $12.35 \pm 0.17$ & 9.158 & 0.000 \\
\hline Activated partial thromboplastin time (APTT), secs & $30.62 \pm 0.55$ & $23.43 \pm 0.47$ & 9.928 & 0.000 \\
\hline Platelets counts $(\mathrm{PLT})\left(\mathrm{x} 10^{9} / \mathrm{L}\right)$ & $228.75 \pm 8.39$ & $240.18 \pm 8.16$ & -0.977 & 0.333 \\
\hline
\end{tabular}

The coagulation related parameters viz: activated partial thromboplastin time and Prothrombin time under investigation showed a decrease after exercise. In the female, the mean prothrombin time was 14.25 and 12.08 
seconds before and after exercise respectively (Table 1). In the male, prothrombin time was 14.29 and 12.35 seconds before and after exercise respectively (Table 2). There was significant different $(\mathrm{P}<0.001)$ before and after exercise. The findings showed that exercise decreases prothrombin time. Furthermore, activated partial thromboplastin time in female was 31.71 and 24.29 seconds before and after exercise respectively (Table 1 ), while male had mean value 30.62 and 23.43 seconds before and after exercise respectively (Table 2). Typically, there was a significant different $(\mathrm{P}<0.001)$ before and after exercise. Abdullah [6] reported that haemostatic factors play essential role in prothrombotic state and in the pathogenesis of cardiovascular diseases. According to El-Sayed [8], short term exercise is frequently linked with a significant shortening of activated partial thromboplastin time and a marked elevation in factor VIII (FVIII). Tripodi et al. [15] reported that a decline in activated partial thromboplastin time could be associated with the risk of venous thromboembolism. Studies are now suggesting that risk of hypercoagulability could be due to short activated partial thromboplastin time [11].

\section{Conclusion}

This study evaluated the effects of exercise on prothrombin time, activated partial thromboplastin time and platelets counts on students from a Nigeria tertiary educational Institution. The study found that there was a significant decline in prothrombin time and activated partial thromboplastin time, and no statistical variation in platelets count before and after the exercise Although, there was a slight increase in platelet count after the rigorous exercise. This suggests that rigorous exercise leads to activation of the coagulation cascade which may lead to hypercoagulability, thereby predisposing the subjects to an increased risk of cardiovascular derangements.

\section{Ethical Consideration}

Permission was obtained from the ethics committees of the Medical Laboratory Science Department of Madonna University, Elele, Nigeria and Federal Medical Centre Yenagoa, Nigeria. Informed consent was obtained from the patients prior to sample collections.

\section{References}

[1] Lekakis, J., Triantafyllidi, H., Galea, V., Koutroumbi, M., Theodoridis, T., Komporozos, C., Ikonomidis, I., Christopoulou-Cokkinou, V. and Kremastinos, D. T. (2008). The immediate effect of aerobic exercise on haemostatic parameters in patients with recently diagnosed mild to moderate essential hypertension. Journal of Thrombosis and Thrombolysis, 25(2): 179-184.
[2] Bhatti, R. and Shaikh, D. M. (2007). The effect of exercise on blood parameters. Pak J Physiol., 3(2): No page no.

[3] Benoni, G., Bellavite, P., Adami, A., Chirumbolo, S., Lippi, G., Brocco, G., Cuzzolin, L. (1995). Effect of acute exercise on some haematological parameters and neutrophil functions in active and inactive subjects. European Journal of Applied Physiology and Occupational Physiology, 70(2): 187-191.

[4] El-Sayed, M., Omar, A. and Lin, X. (2000). Post-exercise Alcohol ingestion perturbs blood haemostasis during recovery. Thrombosis Research, 99: 523-530.

[5] Rostami, H., Aliyev, A., Madatova, V., Rostami, M. and Golchin, M. (2017). The Impact Of Short-time exercise on some of hemostatic factors in different tissues of rats. Advances in Biology \& Earth Sciences, 2(1): 65-72.

[6] Abdullah, W. Z. (2012). Shortened Activated Partial Thromboplastin Time (APTT): A Simple but Important Marker of Hypercoagulable State During Acute Coronary Event, Coronary Artery Disease - New Insights and Novel Approaches, Angelo Squeri (Ed.), ISBN: 978-953-51-0344-8, In Tech.

[7] Moore, S. (2011). Exercise \& Low Platelet Count. $\mathrm{http} / /$ www.livestrong.com/article/127280-foods-increaseblood-platelets/. Accessed August 12, 2017.

[8] El-Sayed, M. S. (1996). Effects of Exercise on Blood Coagulation, Fibrinolysis and Platelet Aggregation. Sports Medicine, 22(5): 282-298.

[9] Khalid, A. and Zafar, L. (2015). Effect of Haemodialysis on Mean Prothrombin Time and Activated Partial Thromboplastin Time in Patients of End Stage Renal Disease. Journal of Rawalpindi Medical College, 19(3): 247-249.

[10] Peterson, P. and Gottfried, E. L. (1982). The effects of inaccurate blood sample volume on prothrombin time (PT) and activated partial thromboplastin time (aPTT). Thromb. Haemost., 47(2): 101-103.

[11] Lippia, G., Salvagnob, G. L., Ippolitoa, L., Franchinic, M. and Favalorod, E. J. (2010). Shortened activated partial thromboplastin time: causes and management. Blood Coagulation and Fibrinolysis, 21: 459-463.

[12] BoneMarrow (2017). Exercising with a Low Platelet Count. http://www.bonemarrowmx.com/exercising-with-a-lowplatelet-count/. Accessed August 12, 2017.

[13] Wang J. S., Jen C. J., Kung H. C., Lin L. J., Hsiue T. R. and Chen H. I. (1994) Different effect of strenuous exercise and moderate exercise on platelet function in men. Circulation, 90(6): 2877-2885.

[14] Wang, J-S., Jen, C. J. and Chen, H. (1995). Effects of Exercise Training and Deconditioning on Platelet Function in Men. Arteriosclerosis, Thrombosis, and Vascular Biology, 15: 16681674.

[15] Tripodi, A., Chantarangkul, V., Martinelli, I., Bucciarelli, P. and Mannucci, P. M. (2004). A shortened activated partial thromboplastin time is associated with the risk of venous thromboembolism. Blood, 104: 3631-3634. 